Revue de l'Institut des langues et cultures

d'Europe, Amérique, Afrique, Asie et Australie

$11 \mid 2009$

Langues \& cultures de spécialité à l'épreuve des médias

\title{
Sur les pas de Rouletabille
}

Représentations du journaliste de presse écrite dans quelques textes de la littérature de jeunesse italienne, française et anglaise des années cinquante à nos jours

\section{Sylvie Martin-Mercier}

\section{OpenEdition}

\section{Journals}

Édition électronique

URL : http://journals.openedition.org/ilcea/260

DOI : 10.4000/ilcea.260

ISSN : 2101-0609

\section{Éditeur}

UGA Éditions/Université Grenoble Alpes

Édition imprimée

ISBN : 978-2-84310-179-3

ISSN : 1639-6073

Référence électronique

Sylvie Martin-Mercier, « Sur les pas de Rouletabille», ILCEA [En ligne], 11 | 2009, mis en ligne le 30 avril 2009, consulté le 08 mars 2021. URL : http://journals.openedition.org/ilcea/260 ; DOI : https://doi.org/ 10.4000/ilcea.260

Ce document a été généré automatiquement le 8 mars 2021

(C) ILCEA 


\section{Sur les pas de Rouletabille}

Représentations du journaliste de presse écrite dans quelques textes de la littérature de jeunesse italienne, française et anglaise des années cinquante à nos jours

\section{Sylvie Martin-Mercier}

1 Dans la seconde moitié du $\mathrm{xx}^{\mathrm{e}}$ siècle, le journaliste, et plus précisément le reporter qui a fait son apparition au début du siècle, occupe une place particulière dans le quotidien et l'imaginaire populaire, au point de devenir un des personnages privilégiés de la " paralittérature ». Pourtant, contrairement aux reporters de bandes dessinées ${ }^{1}$ qui, au moins dans l'aire francophone, se sont parfois installés dans l'imaginaire collectif, dépassant le cadre de leur lectorat réel, relativement rares sont les journalistes jouant un rôle de premier plan dans les autres œuvres de la littérature de jeunesse des années cinquante à nos jours. Toutefois, si les protagonistes, notamment les héros éponymes, sont peu nombreux, plus fréquents sont les seconds rôles ou figurants, aussi cette figure professionnelle est-elle loin d'être insignifiante. De par son âge, le jeune lecteur n'est généralement pas encore un lecteur de presse, si ce n'est de celle qui lui est explicitement destinée, ce qui peut expliquer cette fréquence basse. Le journaliste est un adulte et donc plus difficilement un personnage auquel s'identifier pour l'enfant. Pour ce lecteur particulier, le personnage du journaliste n'est a priori pas porteur des mêmes images, stéréotypes ou préjugés que pour le public adulte dont le regard est en (large) partie influencé par les stéréotypes qui circulent dans la société. Mais l'auteur pour la jeunesse reste un adulte, souvent motivé par des intentions éducatives, parfois cachées derrière l'humour, l'ironie et la mise à distance. Les auteurs vont donc transmettre, à leur jeune lecteur, des images qui peuvent façonner le regard qu'ils porteront sur cette profession, une profession qu'ils ne «connaissent » pas encore. En 1997, Ruth Amossy et Anne Herschberg Pierrot soulignaient la force et l'impact des images transmises aux jeunes :

Les enfants et les adolescents prennent connaissance de certaines réalités à travers les séries télévisées, la B.D., mais aussi les livres scolaires. L'impact de ces représentations s'avère puissant dans le cas non seulement des groupes dont on n'a pas une connaissance effective, mais aussi de ceux qu'on côtoie quotidiennement ou auxquels on appartient. ${ }^{2}$ 
2 Nous pouvons associer à cette liste la littérature de jeunesse, notamment la littérature de grande consommation dont relèvent plusieurs œuvres de notre corpus. Les textes pour la jeunesse peuvent ainsi relayer des stéréotypes existants, contribuer à la création de types ou stéréotypes, ou encore revisiter et détourner des clichés. Ainsi que l'indiquait Ruth Amossy, notre attention doit se porter «[...] non seulement sur la persistance et l'impact des stéréotypes, mais aussi sur leur acquisition par le truchement de l'éducation officielle - les livres scolaires - et des divertissements livres pour la jeunesse, B.D., jouets, télévision... $»^{3}$. Par cette analyse, nous souhaitons explorer les caractéristiques et fonctions des journalistes de presse et de leurs productions dans quelques textes destinés à la jeunesse, pour repérer les messages et images qu'ils véhiculent et déterminer avec quels ressorts ils investissent les univers fictionnels destinés aux enfants. Il s'agira notamment de voir si ces textes diffusent des images stéréotypées des journalistes, tout comme la bande dessinée a pu contribuer à le faire ${ }^{4}$. Une typologie des figures de journalistes et de leurs fonctions puis l'analyse du discours sur les journalistes, leurs méthodes et leurs écrits qui émergent de nos textes, nous amèneront à voir comment on peut trouver à côté d'une représentation « réaliste» de ce thème un traitement plus ou moins fantaisiste mais néanmoins porteur de sens.

3 Nous nous proposons donc de dégager les représentations des journalistes dans un corpus multilingue, principalement italien et français, avec un excursus dans la littérature anglaise. Notre corpus est constitué de textes publiés depuis les années cinquante jusqu'à aujourd'hui, parmi lesquels des romans, policiers notamment, des nouvelles et, de manière plus inattendue, des poésies. ${ }^{5}$ Les textes sont, pour certains, signés par des auteurs reconnus comme faisant partie des plus grands pour la qualité de leur œuvre, accueillis et diffusés par l'école tels Gianni Rodari ${ }^{6}$ pour l'Italie et Yak Rivais ${ }^{7}$ pour la France ; nous pouvons ajouter à ce groupe des auteurs moins connus aux productions originales et de grande qualité littéraire pour leur écriture, tel Claude Bourgeyx ${ }^{8}$. Un autre groupe de textes relève au contraire de la littérature de consommation, de séries à (très) grand succès, même si de natures différentes: Fantômette ${ }^{9}$ de Georges Chaulet ${ }^{10}$ publiée chez Hachette dans la Bibliothèque Rose ${ }^{11}$ pour le secteur français; pour l'Italie, Geronimo Stilton ${ }^{12}$ et quelques volumes tirés d'une collection de petits polars, "I Giallissimi per ragazzi " de l'éditeur Crealibri ${ }^{13}$ et Carlo Ossola $^{14} /$ Harry Potter ${ }^{15}$ de J.-K. Rowlings pour la Grande-Bretagne. Ces textes qui évidemment ne sont pas à mettre sur le même plan nous permettent par leur diversité de composition et de style, d'intention, de public et de diffusion de cerner de manière plus globale les figures de journalistes que les jeunes lecteurs peuvent rencontrer. Nous avons par ailleurs sélectionné des textes où le journaliste oeuvre dans son pays, voire simplement à un niveau local, avec quelques exceptions certes, ce qui exclut le grand reporter ${ }^{16}$.

4 Même si les lecteurs adultes français ont tous à l'esprit Rouletabille ${ }^{17}$, dans la seconde moitié du xxe siècle, le journaliste est rarement protagoniste des aventures destinées aux jeunes lecteurs, cela s'expliquant probablement par le fait qu'il n'appartient pas vraiment au quotidien et à l'imaginaire enfantin ${ }^{18}$. Nous pourrions poser comme hypothèse que le journaliste est d'abord auteur d'articles, or en littérature de jeunesse, comme en littérature générale et surtout en paralittérature, il n'est souvent pas considéré comme un auteur et sa profession devient fréquemment un alibi pour 
d'autres activités. Voyons donc quelle typologie de journalistes se dégage de notre corpus.

Dès les années cinquante, dans ses premières compositions, où sont très présentes les préoccupations sociales et économiques de l'après-guerre mais où pointe déjà la veine humoristique, Gianni Rodari met en scène des journalistes, soit comme protagonistes ou sujets d'un texte, soit comme éléments secondaires et néanmoins moteurs pour la narration, tous faisant l'objet d'un discours critique. Cet écrivain appartient à une lignée d'auteurs italiens pour la jeunesse qui furent journalistes avant d'écrire pour les enfants et ont parfois même mené les deux carrières de front, explorant une profession dont ils connaissent parfaitement les pratiques; il s'inscrit ainsi dans l'héritage de Collodi, De Amicis, Vamba ${ }^{19}$. Point essentiel, il publie ses premiers textes dans des journaux de gauche, dans l'édition du dimanche de l'Unità, destinée à la famille, et des revues s'adressant aux parents et aux pédagogues : contrairement à ce que nous avons posé au départ, il écrit - cas exceptionnel - pour des enfants qui ont un contact précoce avec un journal, orienté politiquement, même si cela se fait par la médiation des parents. De manière particulièrement originale, les journalistes, présents dans plusieurs de ses romans et récits brefs ${ }^{20}$, deviennent chez lui le sujet de poésies et comptines. La poésie «Il giornalista " [ Le journaliste »] s'ouvre ainsi : " Ô journaliste envoyé spécial/quelles nouvelles apportes-tu au journal ? $\star^{21}$. Journaliste professionnel et auteur attentif à la langue et aux tours que peuvent jouer les fautes, Rodari propose ensuite une poésie intitulée «Il refuso » [ « La coquille »] ${ }^{22}$ :

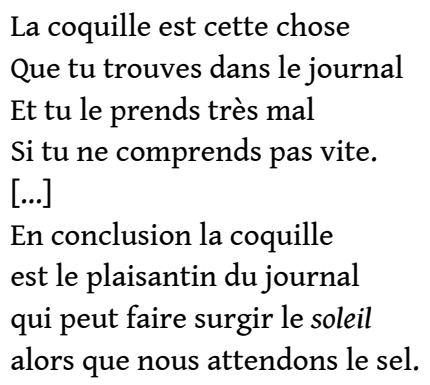

Dans son dernier roman, Il était deux fois le baron Lambert, cet auteur met en scène une véritable cohorte de journalistes, venus des cinq continents sur les bords d'un petit lac piémontais, pour suivre en direct l'occupation de l'île du baron Lambert par 24 bandits :

Journalistes, radios et téléreporters sont arrivés des cinq continents, car le baron Lambert est célèbre du pôle Nord au pôle Sud à cause de ses banques [...] Il y a dans chaque localité des journalistes installés sous les arcades de la place centrale, d'autres perchés sur les balcons et sur les toits. Il y a des longues-vues et des télescopes braqués sur l'île depuis tous les belvédères des routes qui font le tour du lac. Il y a de puissants téléobjectifs constamment en batterie sur les clochers de tous les villages dans un rayon de trente kilomètres. ${ }^{23}$

Rodari nous offre ici une vision digne des scènes de paparazzi. Les actions des journalistes scanderont ce texte. Nous retrouvons le journal et le journaliste dans les mondes fantastiques de Rodari; ainsi dans son bestiaire, les chats, animaux qu'il affectionne particulièrement, ont-ils aussi leur journal : "Les chats ont un journal / Avec toutes les nouvelles / Et sur la dernière page / La 'Petite publicité' 24 . Dans Gelsomino nel paese dei bugiardi ${ }^{25}$, paru en 1971, Gianni Rodari raconte les aventures de Gelsomino, chanteur à la voix extraordinaire mais d'une puissance telle qu'elle brise même les murs les plus résistants. Gelsomino doit alors quitter son village et arrive au pays des menteurs, où le roi, Re Giacomone, a imposé à son peuple de toujours mentir 
ou dire exactement le contraire de ce qu'il pense ou voit. Un journal, Il perfetto bugiardo [Le parfait menteur], et ses journalistes seront les vecteurs de ces mensonges. Si ce texte est écrit plus de 25 ans après la fin de la deuxième guerre mondiale et la chute du fascisme, il n'est pas à exclure que Gianni Rodari ait encore à l'esprit la censure et l'instrumentalisation de la presse, à des fins de propagande, pendant les années de régime fasciste. Autres temps, autre bestiaire sans visée éducative et sociale : le rat Geronimo Stilton dirige l'Echo du Rongeur, parfaitement localisable dans la ville de Souricia grâce aux cartes et dessins qui sont proposés aux lecteurs dans ces livres amplement illustrés, qui ont acquis leur notoriété grâce à leur lecture extrêmement aisée et ludique. Les journalistes n'apparaissent donc pas uniquement dans les récits réalistes et nous les retrouvons dans les plus célèbres histoires de sorciers. Dès le premier tome des aventures de Harry Potter, nous découvrons La Gazette du sorcier, principale source d'information des sorciers avec Le chicaneur ${ }^{26}$. Dans les premières pages des Reliques de la mort ${ }^{27}$, avant de quitter définitivement la maison de Privet Drive, Harry parcourt la pile de journaux arrivés pendant l'été, livrés par hibou, sur abonnement ou payés au numéro. Dans cette série se dégagent deux figures de journalistes, Xenophilius Lovegood et Rita Skeeter qui jouent à un moment particulier des rôles de premier plan. Caractéristique intéressante, ces journalistes font l'objet de descriptions précises dans ce dernier tome ; le premier, Xenophilius Lovegood, est :

[...] un sorcier à l'apparence singulière. Louchant légèrement, ses cheveux blancs semblables à de la barbe à papa lui tombant sur les épaules, il portait une casquette avec un pompon qui pendait devant son nez et une robe d'une couleur jaune d'œuf si vive qu'on en avait les larmes aux yeux. [RM, 143-144]

Et cet accoutrement correspondrait à ses «[...] vues un peu particulières ». Rita Skeeter est longuement interviewée par une autre journaliste, Betty Braithwaite qui, visiblement sous le charme, souligne que : "Lorsqu'on la voit, Rita Skeeter parait beaucoup plus douce et chaleureuse que ne le laisserait supposer la férocité de ses célèbres portraits. » L'interview est accompagnée d'une photo que Harry qualifie de «nauséabonde » avant de décrire ainsi la journaliste :

[...] une femme aux lunettes incrustées de pierreries, avec des cheveux blonds soigneusement bouclés, les dents découvertes en un sourire qu'elle voulait triomphant, et de longs doigts qui ondulaient vers lui. [RM 33]

Les mises de ces journalistes sont bien peu conventionnelles et ne ressemblent guère à l'image stéréotypée du journaliste que nous trouvons dans les textes de fiction. Rita Skeeter et Betty Braithwaite appartiennent de plus aux rares figures féminines liées au monde de la presse. Dans Fantômette, Æil de Lynx, journaliste à France-Flash, correspond bien davantage dans ses traits à l'image que l'on se faisait après-guerre du reporter "pipe au bec et casquette à carreaux sur le crâne » [MIPF 59]. Son rédacteur en chef, Tony Truand, n'échappe pas non plus au cliché en ce qui concerne son apparence, lui qui traverse frénétiquement sa rédaction «[...] en manches de chemise, les lunettes sur le front, un cigare éteint entre les dents » [MIPF 55]. Minaccia alla città ${ }^{28}$ nous transporte dans le New York de la fin des années Trente. Rex Skydog est un chien détective privé, chargé de retrouver une jeune femme disparue, fille d'un richissime banquier, candidat aux élections municipales. Le second chapitre intitulé «Scoop » est tout entier consacré au journaliste Tim «Scoop» Johnson qui sera décrit en ces termes : «Scoop était maigre et sec, tellement grand que les vestes tombaient de ses épaules comme les plis d'une tenture. Il ressemblait à un portemanteau perroquet avec son éternel nœud papillon multicolore. » $[\mathrm{MC} 23]^{29}$ Si le nœud papillon lui confère une 
image respectable, sa mise peu soignée le rapproche des journalistes et détectives de fiction, peu soucieux de leur apparence, entièrement occupés par leur mission. Toutefois, dans nos textes, nombre de journalistes ne font pas l'objet d'une description, même succincte, ce qui tend à prouver qu'en dernière analyse seule leur fonction, leur rôle importent ${ }^{30}$.

10 Tandis que les journalistes présents dans la première catégorie de textes présentés font un travail de journaliste d'information, de reporter, avec un glissement vers la presse à scandale pour skeeter qui tombe dans la dénonciation calomnieuse et Lovegood toujours en quête de complots, dans les romans policiers, ils jouent des rôles plus actifs partageant la vedette avec des détectives. Pour lancer son enquête, Rex Skydog appelle son ami, "reporter spécialiste de faits divers et un de mes grands amis. Nous échangions souvent des informations utiles et intéressantes pour chacun d'entre nous ; chose fort précieuse dans notre profession " [MC 17$]^{31}$. Nous découvrons ainsi les collaborations entre détectives et journalistes, l'un maniant le pistolet, l'autre non, mais tous deux évoluant en marge des règles que doit suivre la police avec laquelle ils collaborent par ailleurs. Étrangement, dans Delitto allippodromo, ce même Tim, journaliste américain spécialiste de faits divers, écrira un article sur les courses hippiques dans un journal parisien ${ }^{32}$. Dans d'autres textes, la profession de journaliste sert aussi essentiellement à justifier l'enquête et à permettre à un personnage de questionner, fureter, interroger, soit en marge des investigations de la police, soit avec l'assentiment de cette dernière. De par sa position, le journaliste apprend forcément des choses et surtout sa profession lui laisse une grande liberté de temps et d'organisation. Le journaliste CEil de Lynx - de son vrai nom Pierre Dupont - a tout le loisir d'accompagner son amie Fantômette, même s'il se fait parfois houspiller par son rédacteur en chef. La collaboration avec Fantômette est équilibrée : tantôt elle le sollicite, lui, l'adulte possédant une voiture, ce qui lui permet de se rendre rapidement en des lieux éloignés, tantôt il propose à la jeune justicière de l'accompagner ou lui demande de l'aider. Ils trouveront en général un accueil favorable auprès de la maréchaussée, grâce à son statut de journaliste: «Le brigadier Jonquille reçut avec empressement ce journaliste venu spécialement de Paris pour enquêter dans la petite ville », pouvons-nous lire dans Fantômette et le brigand. Dans Esprit fantômes, Lucie, journaliste photographe, accompagne son ami Jaz, enquêteur de police, sur les scènes de crime, interrogeant et fouillant, sans que le commissaire ne trouve rien à redire. Ses activités professionnelles sont aussi à la source d'aventures: pour avoir osé photographier pour son journal une idole en Afrique, elle est enlevée et menacée de mort.

11 Contrairement aux journalistes rencontrés chez Harry Potter, la personnalité de Tim suscite un grand respect, à l'image du regard que l'on portait dans les années trente sur cette profession mythifiée :

Tim avait commencé sa carrière comme coursier auprès d'un journal, la Gazette justement. Son intelligence, sa ténacité de dogue et sa ruse de vieux renard chevronné, unies à une grande intuition quand il s'agissait de dénicher les nouvelles les plus sensationnelles en partant de légers indices, l'avaient amené en peu de temps à grandir dans l'estime des propriétaires du journal ; échelon après échelon, il avait connu une carrière extraordinaire et il était monté dans les étages supérieurs de l'immeuble, ceux qui comptent ! Et que cela soit bien clair : seulement et uniquement pour ses mérites.

Son bureau était à côté de celui du directeur. [MC 22] ${ }^{33}$ 
Cette description pourrait en tous points correspondre à celle d'un détective. Il possède de grandes compétences: «Peu de gens connaissaient comme lui les crapuleries et les intrigues qui se cachaient [...].» [MC 21 $]^{34}$. De Tim, qui enquête sur les liens entre la haute finance et le crime organisé, ce qui lui vaut non seulement de recevoir des menaces de mort mais aussi que l'on attente directement à sa vie par un mitraillage en règle de sa voiture, Rex Skydog souligne l'habileté à découvrir des informations ainsi que son courage. Ces journalistes font preuve d'une bravoure extraordinaire : «[...] le risque de se faire seringuer pour l'exactitude de l'information le troublait, sans toutefois l'intimider. » [MC 18] $]^{35}$. Et leur action est qualifiée de véritable croisade : «[...] [sa] très dure bataille qui prenait chaque jour davantage les proportions d'une gigantesque autant qu'impossible croisade contre le crime. $»^{36} \mathrm{Et}$ nous retrouvons ici le stéréotype positif, l'image mythifiée du reporter qui vit ses enquêtes comme des missions. Mais, parfois, ces personnages font preuve d'une bien grande couardise : ainsi lorsque dans Gelsomino Calimero voulut sauter sur les journalistes ceux-ci : « [...] eurent à peine le temps d'enfiler leur stylo-plume dans leur poche et de sauter dans leur voiture.» [GPB 120$]^{37}$, ce qui nous éloigne de l'image classique et valorisante du journaliste prêt à prendre des risques pour découvrir la vérité.

13 Les variations dans la représentation ne tiennent pas aux différences de genre textuel mais à la fonction du personnage. Ce qui compte dans la mise en scène de cette profession dans les œuvres de fiction semble être la liberté de temps et de mouvement qu'elle autorise. D'autre part, la seule présence du journaliste et sa fonction de témoin suffisent à créer l'effet de réel recherché.

14 Les productions des journalistes sont plus souvent des livres que des articles : ainsi l'ouvrage de Rita Skeeter joue-t-il un rôle fondamental dans le dernier tome de Harry Potter. À la tête d'une rédaction sans cesse en ébullition qu'il ne semble guère diriger, aidé de sa sœur Tea Stilton ${ }^{38}$, envoyée spéciale du journal fondé par leur grand-père, Geronimo Stilton n'écrit que des livres, visiblement pas d'articles, et l'essentiel de son activité semble se résumer à écrire son autobiographie aventureuse. Par un effet étonnant de mise en abyme, le livre qu'il commence à écrire ou publie à la fin de ses aventures est justement celui que le lecteur est en train de terminer. Æil de Lynx est aussi feuilletoniste. Tandis que de nombreux journalistes n'écrivent pas et ne semblent même pas avoir besoin de le faire, comme cela est le cas pour Tintin, d'autres au contraire produisent leurs textes avec une extraordinaire rapidité : « une heure plus tard parut une édition extraordinaire du Parfait menteur $~_{39}$, nous dit-on dans Gelsomino tandis que dans les Reliques de la mort nous apprenons que :

Sans nul doute, Skeeter n'a pas perdu de temps. Son livre de neuf cents pages a été achevé quatre semaines seulement après la mort mystérieuse de Dumbledore, au mois de juin dernier. Je lui demande comment elle a pu réaliser cet exploit avec une telle rapidité.

"Oh, lorsqu'on a été journaliste aussi longtemps que moi, travailler dans des délais impossibles devient une seconde nature. Je savais que le monde de la sorcellerie réclamait à grands cris un récit détaillé de toute cette histoire et je voulais être la première à répondre à cette exigence. $"[\mathrm{RM} 33]^{40}$

$15 \mathrm{Au}$ passage, les auteurs écornent la rapidité qui correspondrait à une nécessité (volonté ?) d'être le premier à traiter un sujet et sous-entendent un réel manque de sérieux dans l'investigation, ce qui correspond aussi au stéréotype du reporter prêt à se jeter sur n'importe quel sujet, pour obtenir un scoop à tout prix. 
16 La production de ces journalistes a donc plusieurs statuts : soit elle n'existe pas ou on n'en parle pas, soit elle est annoncée mais on ne la lira pas; dans certains textes au contraire elle est publiée in extenso, telle l'interview de Skeeter, la nécrologie de Dumbledore, ou par bribes. Elle est donc soit un élément inconsistant, sans aucune utilité, soit un élément d'aboutissement, une finalité. Elle peut être un point de départ, un élément déclencheur ou encore un fil conducteur, cela arrive fréquemment dans la série Fantômette où la lecture du journal peut motiver le démarrage de l'enquête. Dans Senso di giustizia, les textes parus dans les quotidiens ont un retentissement sur la manière de conduire l'enquête, soulignant le rôle que la presse peut avoir non seulement dans la formation de l'opinion mais aussi sur les institutions, en l'occurrence la justice. L'intérêt variable des médias pour les délits amène le magistrat instructeur à accélérer et rediriger son enquête. Après le premier homicide d'un publicitaire, dont la vie était pour le moins plate, "décevante du point de vue journalistique $~^{41}$, l'enquête piétine, les avis de la presse et conclusions de la police se superposant ou se répondant : ni les enquêteurs, ni la presse ne pensent que l'envie soit à l'origine de ce meurtre et la police ne commente pas les hypothèses émises par la presse. Parallèlement, presse et police cessent de s'intéresser à l'affaire: "L'enquête s'étira vers un classement probable. Dans les journaux, comme d'habitude, les articles se firent plus courts dans les pages intérieures, jour après jour, jusqu'à disparaître. $»^{42}[S G 6]$. Après le second homicide, l'enquête prend un nouveau tour, uniquement parce que les journaux ont beaucoup parlé de l'affaire, agitant le spectre des années de plomb. Le troisième meurtre est celui d'un journaliste, rédacteur en chef d'un quotidien milanais, et survient en pleine crise de gouvernementale, ce qui aurait dû reléguer la nouvelle dans les pages internes des journaux. Or, vu la victime, elle revient en première page. Le magistrat chargé de l'enquête arrête même de lire les journaux. Au fur et à mesure, le texte précise l'évolution des articles, leur place, leur contenu, les personnes interviewées, les changements dans la teneur des titres. Ici, les articles ne sont pas signés, car c'est uniquement leur contenu qui compte, leur rôle dans l'histoire et leur fonction dans la narration. Par ailleurs, les extraits et discours tronqués cités dans le texte apportant des informations réduites au strict nécessaire, correspondent à une recherche fonctionnelle d'efficacité narrative mais nous ôtent toute vision globale du texte journalistique. L'article et non seulement l'activité du journaliste devient élément déclencheur de l'action (principale ou secondaire). Dans les premières pages des Reliques de la mort, Harry Potter trie les journaux, à la recherche d'un numéro qui présentait « en première page une brève annonce de la démission de Charity Burbage, le professeur d'étude des Moldus à Poudlard. » Nous pensons donc que c'est cette nouvelle qui l'intéresse or :

Il trouva enfin le journal. L'ouvrant à la page 10, il s'enfonça dans le fauteuil de son bureau et relut l'article qui l'intéressait.

EN SOUVENIR D'ALBUS DUMBLEDORE

par Elphias Doge [RM 27]

Suit un long article nécrologique de quatre pages, qui n'est pas rédigé par un journaliste mais par un ami du défunt et va constituer un des ressorts dramatiques de ce dernier volume : on y annonce que le père de Dumbledore, le directeur de Poudlard tant admiré par Harry, avait été condamné pour le meurtre de trois Moldus, que Dumbledore était très lié à Grindelwald, lorsque sa sœur Ariana mourut dans des circonstances non élucidées. Cet article provoque un véritable choc pour Harry qui «[...] pensait connaitre bien Dumbledore mais, après avoir lu l'article, il était forcé 
d'admettre qu'il le connaissait à peine. » [RM 81] et va induire une série d'actions ou de réactions, relancées par les annonces; en jetant un coup d'œil à La Gazette du jour :

En bas de page figurait un titre plus petit que la manchette, au-dessus d'une photo représentant Dumbledore marchant à grands pas d'un air tourmenté :

DUMBLEDORE : ENFIN LA VÉRITÉ?

À ne pas manquer la semaine prochaine: l'histoire scandaleuse d'un génie imparfait que

beaucoup considèrent comme le plus grand sorcier de sa génération. [RM 32]

Ce titre et cette annonce correspondent aux formules dont sont friands les périodiques de la presse populaire et à scandale, anglaise notamment. Nous retrouvons ce type de titre avec đil de Lynx qui écrit non pas pour la presse à scandale mais pour un journal à grand tirage qui s'intéresse essentiellement aux faits divers et aux célébrités. Ce journaliste utilisera aussi les possibilités que lui offre la presse pour capturer un brigand : il n'hésitera pas à signer un article provocateur, "explosif » à l'encontre du bandit Mandrin pour réussir à le rencontrer [FB 65]. Cet article, intitulé « Mandrin n'est qu'un gredin ", nous est donné à lire en partie et provoque l'enlèvement du journaliste mais aussi une augmentation extraordinaire des ventes du journal. Nous voyons apparaître ainsi l'image du journaliste prêt à courir les plus grands risques, pour faire appréhender certes un voleur mais aussi pour « faire un papier sensationnel » [FB 84] qui lui offrira les honneurs de la Une. Eil de Lynx est d'ailleurs le seul reporter du corpus à rechercher scoops et articles susceptibles de le rendre célèbre et de lui valoir quelque cadeau, une nouvelle pipe, une augmentation pour s'offrir une nouvelle voiture. Il semble aussi être le seul confronté à la recherche du sujet, aux difficultés d'écriture et à la hiérarchie : de son rédacteur en chef, exigeant, colérique, pensant surtout à la Une, à son président-inspecteur général, M. Lhénorme, un petit homme très affairé, extrêmement étourdi mais qui consent généralement à financer ses voyages, si le journal peut en tirer quelque avantage. đil de Lynx est ainsi le journaliste le plus proche du stéréotype forgé à partir de l'image de Rouletabille, comme lui constamment happé par le désir d'enquêter et systématiquement rappelé à la nécessité d'écrire et nous pourrions transposer sur lui cette citation de Jacques Dubois à propos de Rouletabille :

[...] Rouletabille conjoint subtilement en son statut de reporter l'enquêteur ou le détective (pour le côté droit) avec le publiciste ou le journaliste (pour le côté lettres). Ce qui lui vaut une position incertaine, tiraillée entre profession et vocation, entre travail aliéné et œuvre libre. ${ }^{43}$

Ces journalistes sont rarement décrits pendant qu'ils écrivent : Tim accueille Rex avec ces mots : «Salut, Rex, j'étais juste sur le point d'envoyer sous presse l'édition du soir ; je n'ai pas le temps maintenant. $»^{44}$ Il dicte ses articles et semble enquêter essentiellement par téléphone, on ne fait allusion ni à des archives, ni à des papiers : "Son bureau était uniquement occupé par trois téléphones, avec lesquels il dictait ses articles et accomplissait la plus grande partie de son travail, qui consistait au final à établir des contacts avec la moitié de la planète. $»^{45}$ [MC 22-23], ce qui souligne ses capacités intellectuelles de déduction et d'investigation. ๔iil-de-Lynx, qui est au final une des figures les plus construites que nous rencontrions dans nos textes, dessinée par touches au fil des histoires, est vu prenant des notes et parle à plusieurs reprises de son futur article pour France-Flash, en général en lien avec la résolution de l'affaire d'enlèvement qui les occupe mais pas systématiquement. Æil de Lynx sera plus fréquemment décrit à sa table de travail dans la salle de rédaction, mais aussi - et cela est plus original - en train de réfléchir à la structure et au contenu de ses articles. 

le sera que de manière exceptionnelle :

[...] j'aimais sa façon de travailler : les articles qu'il écrivait faisaient plus de mal aux délinquants de tout poil qu'une décharge de chevrotine. Il avait, à mon avis, beaucoup de courage; eh oui, car, si vous l'ignorez, les batailles ne se gagnent pas seulement avec les pistolets, mais il faut aussi un bon cerveau $!^{46}$

21 résultat de leur enquête qui prévaut et le contenu de l'article se confond avec les résultats de l'enquête :

Tim, surnommé Scoop pour son habileté à dénicher les informations les plus secrètes, avait publié une série d'articles dans la Gazette, le quotidien le plus indépendant et le plus vendu de toute la ville, dénonçant les liens possibles entre la pègre, le monde des affaires et la politique.

Certes il savait vraiment de très nombreuses choses sur notre très chère et très sale métropole et ses affaires louches... Mais ce qui inquiétait c'était peut-être qu'il ne disait ni n'écrivait nombre de ces choses ! ${ }^{47}$

Avec un humour cinglant, Rodari insiste fréquemment sur l'inconsistance ou le caractère mécanique, attendu, stéréotypé d'une certaine écriture journalistique :

Des milliers de journalistes tapaient à la machine le début de leur article de fond :

Sur le visage souriant de Jip, écrivaient quatre-vingt-quinze pour cent des journalistes, nous avons lu un souhait de paix et de joie pour notre vieille planète.

Quand aux cinq journalistes qui manquaient pour faire cent pour cent, ils écrivaient la même phrase, mais au lieu du mot souriant, ils mettaient l'adjectif joyeux. [JT 78-79]

Il faut aussi souligner le choix fréquent d'insérer l'article, avec ses caractéristiques typographiques particulières, dans le flux du texte et cela non seulement dans les ouvrages illustrés. Le texte acquiert ainsi non seulement une mise en évidence mais aussi une matérialité qui en font un «vrai » article, participant ainsi à l'effet de réel. Par contre, à plusieurs reprises, nous découvrons la teneur des papiers d'œil de Lynx alors qu'il les dicte au téléphone, ce qui se justifie par son éloignement de Paris lorsqu'il part enquêter en France ou même à l'étranger. Pendant les années soixante-dix, le téléphone était le moyen de communication le plus rapide et efficace : cette stratégie de présentation a l'avantage de mettre en scène le journaliste dans son activité mais transforme le texte écrit en texte raconté.

Les journalistes peuvent aussi prendre directement part à l'action, ce qui peut être contestable du point de vue déontologique. Quand, dans Il était deux fois le baron Lambert, Duilio doit trouver trente kilos de scotch, les journalistes les plus jeunes décident de s'en charger et tout à coup le rythme s'accélère :

- Nous allons nous en occuper! crient les jeunes journalistes; et de fait ils se répartissent en équipes, bondissent dans leurs voitures et se mettent à ratisser villes et villages de la région, raflant tout le scotch disponible. Au bout d'une heure, ils reviennent avec des montagnes de rouleaux de toutes les couleurs, qu'ils remettent à Duilio avec la légitime fierté que l'on peut éprouver quand on a conscience de participer à une entreprise historique.

- Moi je l'ai pris bleu, parce que ça va bien avec la couleur du lac.

- Tenez, trente kilos de scotch offerts par la Gazette de Quarna!

- Trois kilos et demi au nom du Courrier de la Valstrona! [BL 136] 
lorsqu'ils font leur travail, ils s'attaquent souvent à des sujets futiles et produisent des écrits qui pourraient nous paraître parfaitement inutiles. Même Æiil de Lynx s'appuie sur les lectures de Fantômette pour étayer son article sur le nouveau Mandrin, dans Fantômette et le brigand. Avec des techniques narratives similaires, Rodari et Yak Rivais soulignent les travers des journalistes telles la faiblesse et incohérence de leurs questions. Dans Il était deux fois le baron Lambert, Rodari s'attarde longuement pour le plus grand plaisir du lecteur sur le travail des reporters :

D'ordinaire il arrive à temps pour la conférence de presse du batelier Duilio.

- Qu'avez-vous acheté aujourd'hui?

- Douze poulets, sept lapins, des pâtes, du riz, cinq variétés de fromage, trente kilos

de fruits, du café, du sucre, du sel.

- Combien de sel?

- Deux paquets de sel fin et deux de gros sel.

Quand Duilio remonte dans sa barque pour transporter le ravitaillement sur l'île, il est salué par de grands applaudissements et les photographes lui crient :

- Regarde par ici, Duilio ! Souris ! Lève bien haut ce régime de bananes.

Les photographes tutoient tout le monde.

$\mathrm{Au}$ retour Duilio est suivi d'un cortège de barques bourrées de journalistes, qui posent des questions et prennent des notes :

- Qu'ont dit les bandits?

- Avez-vous vu le baron?

- Avez-vous vu Monsieur Alfred?

- Avez-vous fait le service militaire?

- À quel âge vous êtes-vous marié ?

- Combien d'enfants avez-vous?

- Combien de litres de vin buvez-vous par jour?

Les journalistes, eux, le vouvoient toujours. [BL 79-80]

Avec des questions tout aussi incohérentes, ils n'hésitent pas à interviewer les petitsenfants du batelier qui ne jouent absolument aucun rôle dans cette affaire d'enlèvement : 
Eux aussi sont harponnés par les journalistes et interviewés par la télévision :

- Qu'est-ce que tu préfères, Zorro ou Superman?

- Tu es plus fort en cybernétique ou en anthropologie structurale?

- Combien ça fait trois fois huit vingt-quatre ? [BL 82]

L'absence fréquente de réponse souligne de manière appuyée et caricaturale que ces journalistes n'écoutent ou n'entendent pas les réponses, voire ne s'en préoccupent même pas. Les précisions inutiles, par exemple à propos de la quantité de sel achetée, accentuent l'effet parodique ou caricatural. Nous retrouvons les figures d'accumulation chez Rivais. "L'enfant qui flottait sur l'eau » [P. 99-114] narre l'histoire de Julien qui marche, glisse et s'assied sur l'eau. Le maitre nageur prend des photos que Monsieur Bertrand, le journaliste fait paraître dans le journal provoquant la survenue massive de journalistes :

Les journalistes happèrent l'enfant dès qu'il parut :

«Est-il exact que tu marches SuR l'eau?»

«Est-il exact que tu peux y faire des galipettes?»

« Est-ce que tes vêtements rétrécissent au lavage? » [P. 105]

Les questions posées sont absolument hors de propos et même tendancieuses ou d'une stupidité extrême ; certes, il s'agit ici d'amuser le lecteur mais aussi de dénoncer les dérapages d'une certaine presse, prête à tout pour faire un papier et vendre un journal :

Une nuée de journalistes s'abattit sur l'enfant à onze heures et demie. Tout le monde l'agrippait, lui collait un micro sous le nez pour le faire parler de n'importe quoi.

«Es-tu catholique, musulman, bouddhiste, israélite, ou animiste?»

«Comment fais-tu pour te laver les pieds?»

«Est-ce que ta grand-mère fait du vélo?»

Julien se débattait. [P. 107]

Mais dans Il était deux fois le baron Lambert, nous assistons aussi à un glissement, avec l'apparition d'un sentiment d'empathie entre les journalistes qui vivent là depuis plusieurs jours et ce pauvre batelier :

- Où allez-vous?

- Charon, un sourire pour la presse

- Comment vont vos petits-enfants? Et votre belle-mère a-t-elle toujours mal au ventre?

Si cela nous fait sourire, et donne une certaine humanité à ces journalistes, nous pouvons imaginer que l'objectivité avec laquelle ils sont censés aborder leur sujet pourrait être mise à mal. Avec beaucoup d'humour, Rodari critique nettement ces personnages dont les techniques d'investigation laissent pour le moins perplexes :

L'île est entourée d'un cordon serré d'embarcations chargées de policiers qui surveillent les bandits. Autour de ce premier cercle il y en a un second de barques bourrées de touristes et d'envoyés spéciaux qui surveillent la police. Sur toute la surface $\mathrm{du}$ lac, par tous les temps, d'autres professionnels ou amateurs de l'information vont et viennent en canot à moteur ou en profitent pour se consacrer au sport de la voile. [BL 75]

Cette critique est accentuée par l'énumération des lieux où se sont postés ces journalistes, positions qui ne permettent pas forcément de voir ce qui se passe mais offrent d'autres plaisirs.

Parmi les observatoires les plus recherchés par la presse, on peut citer :

Le belvédère de Quarna, où la bière est toujours fraîche ;

Le sanctuaire de la Vierge au rocher, à pic sur le lac ;

Une auberge de la Valstrona, d'où l'on ne voit rien mais où l'on mange une 
excellente polenta au civet de lapin ;

La tour de Buccione, construite au XII ${ }^{\mathrm{e}}$ siècle mais toujours solide au poste ;

Le couvent du Mont-Mesma, où les moines recueillent ingénieusement l'eau de pluie mais offrent à leurs hôtes un savoureux petit vin ;

Et, naturellement, juste derrière Orta, à l'endroit le plus élevé du promontoire, l'esplanade du Mont-Sacré [...].

Ces journalistes nous apparaissent comme bien peu intéressés par leur travail, blasés, à tel point qu'ils ratent l'information fondamentale quand les bandits s'envolent en ballon à la faveur de la nuit :

À un certain moment un journaliste en faction sur la place d'Orta croit apercevoir sur le toit de la villa une grande ombre noire. Mais il est si jeune que personne ne le croit. Ses collègues plus âgés ne mettent même pas le nez hors du café où ils sont terrés pour jouer au poker.

Une ombre noire, hein? C'est sûrement le diable.

As-tu remarqué s'il avait des cornes?

Tu n'as pas senti une odeur de soufre?

Peu après, même le journaliste ne voit plus l'ombre noire. [BL 140]

Rodari égratigne à plusieurs reprises la vieille génération des journalistes, qui se moquent des plus jeunes qu'ils jugent incompétents. Et quand les jeunes sont déjà au travail, les plus âgés s'attardent au bord du lac : «Charon débarque lestement et court vers la place centrale, poursuivi par les journalistes les plus jeunes (les plus vieux sont encore en train de déjeuner sur les terrasses des hôtels). » Cas extrême, dans un texte de Claude Bourgey $\mathrm{x}^{48}$, un journaliste apprend par une dépêche que l'immeuble où il travaille brûle : après avoir vérifié par la fenêtre que l'immeuble brûlait bien et appris qu'il fait partie des rescapés, il s'en retourne tranquillement à son article sur le temps qu'il faisait la veille!

Ces journalistes ont parfois des méthodes pour le moins contestables: lorsque Braithwaite demande à Rita Skeeter si elle est restée en relation avec Potter « dont elle a publié une si célèbre interview l'an dernier », voici la réponse qu'elle obtient :

«Oh, oui, nous sommes devenus très proches, répond-elle. Le malheureux Potter n'a pas beaucoup de vrais amis et nous nous sommes rencontrés à l'un des moments les plus déterminants de sa vie: le Tournoi des Trois Sorciers. Je suis sans doute l'une des rares personnes vivantes qui puisse affirmer qu'elle connaît le véritable Potter. » [RM 36]

alors que le lecteur sait pertinemment qu'Harry abhorre cette personne. Elle balaye avec des éclats de rire les réserves apportées par E. Doge «le livre de Skeeter contient moins de faits réels qu'une carte de Choco-grenouille» et, dans le seul but évident de le discréditer, elle affirme, pure invention, que lorsqu'elle l'a interviewé quelques années plus tôt « [c]omplètement gâteux, il semblait penser que nous étions assis au fond du lac Windermere et me répétait sans cesse de faire attention aux truites. » [RM 33-34]

Le travers le plus régulièrement représenté est le harcèlement. Par le biais de l'humour et de la parodie, Yak Rivais dénonce ainsi avec férocité l'acharnement des médias. Dans «L'enfant qui flottait sur l'eau», comme dans les textes de Rodari, travail du journaliste et intervention du photographe proche du paparazzi s'entremêlent :

À la sortie, plusieurs autres journalistes attendaient l'enfant dans la rue. Ils étaient armés d'appareils photographiques et de magnétophones.

Ils demandaient : « Lequel c'est, le barboteur?»

Ils croyaient que les photos étaient truquées et disaient d'un air soupçonneux :

«Les photos, c'étaient des montages? » [P. 103] 
écorne davantage l'image des journalistes de télévision, mais dans cette mêlée, il est difficile de distinguer qui finit par enlever l'enfant: «Et hop! Ils kidnappèrent l'enfant. Le malheureux était étourdi parce qu'ils le tiraient à hue et à dia. Ils le firent monter en automobile et l'emmenèrent. » [P. 105] et devant les protestations de Julien les journalistes font preuve de bien peu de bon sens : «Tu iras après ! » répondaient les journalistes. «L'information d'abord! » Mais l'enfant s'obstine et leur attitude devient ridicule, cynique, montrant leur grande immaturité tandis que le bon sens se trouve du côté de l'enfant :

Les journalistes protestaient; ils disaient que Julien avait tout le temps d'aller s'em***** à l'école, qu'il avait toute la vie devant lui! Ils disaient que, puisqu'il allait devenir célèbre il n'avait plus besoin d'apprendre quoi que ce soit! « Quand on est célèbre ", ricanaient-ils, « on sait tout même quand on ne sait rien ! »

Un nouveau degré est franchi quand ils en viennent à la brutalité verbale, expression métaphorique d'une brutalité physique, déjà extrême si on pense qu'ils ont enlevé l'enfant :

Alors les journalistes le supplièrent et le menacèrent en même temps. " Tu veux mon pied au ${ }^{* * *}$ garnement? Une bonne paire de baffes lui ferait du bien! Est-ce que nous allons à l'école nous autres? » [P. 106]

Alors qu'il s'enfuit, ils le poursuivent, lui promettant de le ramener à l'école sans jamais respecter leur promesse. Ils créent une situation de tension absolument insoutenable et inadmissible vis-à-vis d'un enfant : «'Encore! Encore!' hurlaient les journalistes et les photographes » et ne baissent jamais la garde à tel point que les parents doivent ruser pour l'emmener en classe. Julien finit par prendre les pilules censées le guérir et l'effet est immédiat : «À la fin de la semaine, les journaux l'avaient oublié, la radio diffusait des chansons, la télévision des feuilletons. » [P. 113]. Nous voyons ici l'inconstance des journaux, qui cessent bien vite de s'intéresser à une affaire, attitude que nous rencontrons aussi dans Senso di giustizia.

Rita Skeeter :

«Cette femme, ou plutôt ce vautour devrait-on dire, m'a littéralement harcelé pour que j'accepte de lui parler. J'ai honte de vous avouer que je suis devenu assez discourtois, je l'ai traitée de vieille truie fouineuse, ce qui s'est traduit, comme vous l'avez lu, par des calomnies concernant ma santé mentale. »

Les mêmes techniques d'investigation peuvent être utilisées de manière utile ou néfaste : Scoop parle souvent avec les barmen "pour débusquer les nouvelles les plus étranges, en utilisant sa sympathie et quelques dollars qui changeaient de main. $»^{49}[\mathrm{MC}$ 21] ce qui lui permet de combattre le crime. Lorsque l'on fait remarquer à Skeeter que les «accusations d'inexactitude " qui ont été portées à son encontre ont trouvé un écho, voici sa réponse :

Ma chère amie, répond Skeeter, rayonnante, en me tapotant affectueusement la main, vous savez aussi bien que moi combien on peut rassembler d'informations grâce à un gros sac de Gallions, un refus systématique de s'entendre dire non et une bonne Plume à Papote bien aiguisée ! [RM 84]

Tout comme pour Tim, cet argent ou ces techniques peu avouables permettent d'avoir accès à des sources inédites : «[...] j’ai eu accès à une source pour laquelle la plupart des journalistes seraient prêts à donner leur baguette, quelqu'un qui n'a jamais parlé publiquement jusqu'à maintenant [...]. » Pourtant les objectifs sont nettement moins 
avouables. Tim aussi entretient des relations avec des personnages influents ou susceptibles de lui fournir des renseignements :

Il était aidé par son répertoire téléphonique ultra-secret, introuvable et invisible, son instrument de travail le plus précieux. On racontait qu'y étaient notés les numéros de téléphone personnels des personnages les plus puissants du pays $!^{50}$ [MC 22-23]

Mais d'un journaliste à l'autre les objectifs diffèrent. Dans « L'enfant qui savait marcher sur l'eau ", les journalistes osent proposer «des bonbons et de l'argent » au petit garçon dans une énième tentative pour l'obliger à répondre à leurs requêtes. L'image $\mathrm{du}$ journaliste ne correspond plus à l'image positive de l'enquêteur, du reporter dont le travail perce les travers de la société. Au contraire, ces scribouillards sont à la solde d'une presse uniquement fondée sur le sensationnel, le fait monté en épingle pour être oublié dès qu'un nouvel événement se produit.

Nos auteurs n'hésitent pas à dénoncer la collusion qui peut exister entre journalistes et pouvoir et à stigmatiser les journalistes à la solde du pouvoir. Dans Gelsomino, puisque tous doivent mentir, les contenus des articles de journaux doivent être renversés. Un chapitre est ainsi consacré au journal de cet étrange pays, avec des extraits d'articles :

Le journal était intitulé : Le parfait menteur, et naturellement il était entièrement composé de fausses nouvelles ou de faits réels racontés à l'envers.

Il y avait par exemple un article intitulé: "Une grande victoire du coureur Persichetti ». Voici le texte de la nouvelle : «Le célèbre champion de course en sac Flavio Persichetti a gagné hier la dixième étape du tour du Royaume, en devançant de vingt minutes Romolo Baroni, arrivé second, et de trente minutes et quinze secondes Piero Clementini arrivé troisième. Le peloton, arrivé une heure après le vainqueur, a été battu par Pasqualino Balsimelli qui s'était échappé. ${ }^{51}$ [GPB 71]

En réalité, aucune course n'a jamais eu lieu, mais chaque année le journal organise une fausse course, qui est un appel à contribution financière : les personnages influents, orgueilleux ou à la recherche de publicité, versent des sommes d'argent plus ou moins importantes et sont cités avec plus ou moins d'éloges dans les compte-rendus de cette course imaginaire. Le plus généreux est déclaré vainqueur de l'étape. Et lorsque le journal estime que les dons n'ont pas été assez élevés, il se venge par des commentaires peu élégants. Les liens entre personnages appartenant au monde de la politique et de la finance, pouvoir et médias sont soulignés et non sans rapport avec l'Italie de l'aprèsguerre mais peut-être aussi de la période fasciste.

Mais un autre traitement journalistique est plus inquiétant; on en vient à cacher des événements tragiques en les transformant en bonnes nouvelles : "Tragédie évitée sur la via Cornelia - Cinq personnes ne meurent pas et dix autres n'ont reporté aucune blessure. $»^{52}$ Il s'agit là d'une nouvelle non pas fausse mais retournée. De même annonce-t-on que le concert de Gelsomino n'a pas provoqué le moindre dégât alors que, sous une photographie du théâtre en ruine, on précise « ainsi que le lecteur peut le voir de ses oreilles, absolument rien n'est advenu. ${ }^{53}$ Zoppino est heureusement un lecteur attentif qui, tout en bas de la dernière page, découvre deux entre-filets, intitulés "Smentita », c'est-à-dire "démenti ». Le premier, signé par le chef de la police, concerne tante Panocchia et sa nièce Romoletta, deux personnages importants de ce récit, dont on dit qu'elles n'ont pas été arrêtées et ne sont pas enfermées à l'asile de fous. Le second avis concerne Gelsomino :

Il n'est absolument pas vrai que la police est en train de rechercher le célèbre ténor Gelsomino. Il n'y aurait aucune raison à cela, car Gelsomino ne doit pas du tout répondre des dégâts qu'il n'a pas causés au Théâtre Municipal. Donc, quiconque 
saurait où se cache Gelsomino ne doit pas aller le dire à la police car il serait sévèrement réprimandé. [GPB 74] $]^{54}$

\section{transmission des informations au service de régimes autoritaires et violents. La Gazette} $d u$ sorcier publie en première page l'avis de recherche concernant Harry :

Une immense photo de lui s'étalait à la une. Il lut la manchette qui figurait au-

dessus :

RECHERCHÉ POUR INTERROGATOIRE

DANS L'ENQUÊTE SUR

LA MORT D'ALBUS DUMBLEDORE [RM 32]

Car le pouvoir contrôle la presse, en tout cas Harry est persuadé que le ministère fait pression sur La Gazette pour taire les nouvelles concernant le Seigneur des Ténèbres. Et même dans Fantastique Fantômette, le journal Synovie-Matin se fait-il le relais du culte de la personnalité du Premier Ministre.

Après que Gelsomino a provoqué la destruction de l'asile et la fuite des surveillants et directeur grâce à son chant, les journalistes n'arrivent pas à l'approcher et doivent se contenter d'interviewer un autre personnage qui ne répond pas à leurs questions, nous offrant un échange surréaliste :

- Vous n'avez rien à déclarer au Parfait menteur? lui demandèrent les journalistes.

- Miaou, répondit Calimero en tournant le dos.

- Excellent, dirent les journalistes, vous êtes l'un des témoins oculaires. Pouvezvous nous dire comment il se fait qu'il ne s'est rien passé?

- Miaou, dit encore Calimero.

- Très bien. Nous démentirons de la manière la plus absolue que l'asile se soit écroulé et que les fous se soient dispersés dans la ville.

- Mais voulez-vous bien comprendre, s'énerva Calimero. Voulez-vous bien comprendre, oui ou non, que je suis un chat?

- Vous voulez dire un chien, vu que vous miaulez. ${ }^{55}$

Ici sont montrés du doigt les journalistes qui ne savent pas choisir leurs témoins et ont des idées tellement préconçues que, quelles que soient les déclarations de ces derniers, ils arrivent aux conclusions qu'ils souhaitent, taisant la vérité. Ainsi se pose le problème de la fiabilité des sources, bien souligné dans cette conversation entre Muriel et Doge :

Je crois que Bathilda a tout raconté à Rita Skeeter. Ces allusions, dans l'interview de Skeeter, à une source bien informée, proche des Dumbledore... [...]

- Bathilda n'aurait jamais parlé à Rita Skeeter, murmura Doge.

- Complètement gaga depuis quelque temps, d'après ce qu'on m'a dit, ajouta joyeusement la tante Muriel.

- Si c'est vrai, c'est encore plus déshonorant de la part de Skeeter d'en avoir profité, trancha Doge, et on ne peut accorder aucun crédit à ce que Bathilda a pu déclarer ! [RM 174]

Ainsi faut-il parfois se méfier des journalistes et ne pas leur fournir d'informations et aussi, bien évidemment, se méfier des informations qu'ils peuvent colporter. Pour sa propre sécurité, on essaie de se protéger, en leur cachant volontairement des informations. Dans Les aventures de Jos Tempête. La machination du Scorpion noir, Jos est devenu propriétaire du « Parc olympique » transformé en forteresse inexpugnable car de nombreuses personnes veulent le tuer alors : « aucun visiteur, aucun journaliste n'y avait jamais été admis. ${ }^{56} \mathrm{Et}$ aux journalistes qui ne pourraient être que sceptiques, on ne dit pas tout : sa fille Iliade a les cheveux et la peau naturellement violets comme sa mère, elles sont les deux seules sur terre dans ce cas : 
Les médias ne savaient pas grand-chose à propos de Keridwen. Ils ignoraient, par exemple, que la couleur de sa peau était naturelle.

Si on leur avait appris qu'elle venait d'une autre planète, aucun journaliste ne l'aurait cru. C'était pourtant la vérité. ${ }^{57}$

Dans un bel effet de retournement, Rodari invite quant à lui son jeune lecteur à se méfier des informations qui lui sont données, mais aussi à se méfier de qui se méfie des journalistes! Ce qui atteste de la grande confiance que Rodari a dans ses lecteurs, capables de comprendre cela. Adoptant une attitude pédagogique, il leur donne ainsi les clés pour décrypter les articles. Lisons Jip dans le téléviseur ${ }^{58}$, publié en 1976. Jip est un petit garçon qui a la mauvaise habitude de trop regarder la télévision : il finit par être happé par le téléviseur et naviguer, d'une chaîne à une autre, à travers le monde entier. Cet événement suscite de nombreux commentaires :

- Mais il y a déjà eu un cas d'attraction par la télévision, insistait le comptable Binda; je l'ai lu dans un article d'un certain Rodari. À cette occasion les médecins ont même donné un nom à cette maladie. Si je me souviens bien, ils l'ont appelée la télévisionnite.

- Des inventions, mon cher. Des inventions de la presse, des exagérations. Parfois les journalistes, pour se mettre en valeur, ne mesurent pas leurs mots.

Le commissaire confirma cet important jugement du fonctionnaire en rapportant le cas d'un journaliste qui avait décrit par le détail le vol de la tour penchée de Pise.

- Vous comprenez! Il a inventé qu'elle avait été emportée, morceau par morceau, par une bande spécialisée dans le vol des monuments historiques. Il fut facile de montrer que la nouvelle était fausse et tendancieuse, en présentant au public une simple carte postale illustrée, d'où il résultait que la tour de Pise était toujours à sa place. Mais en attendant le bruit s'était répandu et personne n'épargnait la police dans ses critiques. [JT 59-60]

Cet extrait montre que la presse peut dire la vérité mais que le pouvoir en place et la police, d'une seule et même voix, peuvent occulter cette vérité. Rodari cherche toujours à donner de la situation une vision qui lui semble équilibrée. Le lendemain de cette conversation, les journaux suscitent l'affolement :

Les premières pages étaient noires de titres effrayants :

Jip, reviens

Sur ton antenne!

L'écran rendra-t-il sa proie?

Un enfant de 8 ans

Avalé par son téléviseur

Crime atroce sur la $1^{\text {re }}$ chaîne

Une bande de télévoleurs terrorise la ville

Ici Rodari dénonce la tendance fréquente à recourir à des titres racoleurs, utilisés sans aucun discernement alors qu'ils sont susceptibles de provoquer une panique générale. Il insiste ensuite sur le manque de professionnalisme dans la vérification des sources, repérable dans les multiples versions différentes données de l'événement:

Les articles étaient encore moins encourageants que les titres. L'événement y était raconté de cent façons diverses. Jip y était décrit tantôt comme « un poupon blond à l'air angélique ", tantôt comme "un garnement qui ne laissait pas en paix les sonnettes des portes voisines». Un journal insinuait que l'avocat Prosperi devait savoir quelque chose : après tout, le téléviseur du crime était à lui. D'après un autre journal la chute dans le téléviseur n'était qu'une invention de la police : en réalité Jip avait été enlevé par des êtres ultra-terrestres, probablement des martiens, extrêmement dangereux parce qu'invisibles.

- Invisibles ! grommela Mademoiselle Emma, en jetant les journaux dans la poubelle. Je voudrais que tous les journalistes deviennent vraiment invisibles. 
Les journaux du soir publièrent eux aussi de sensationnelles informations sur Jip. Mais il n'était que trop évident qu'il s'agissait de « fausses nouvelles » : il n'y avait pas deux journaux qui disaient la même chose. [Jip 61-62] porteur d'optimisme, de rêve et même de magie. Dans l'Italie de l'après-guerre, en pleine guerre froide, Rodari, membre du PCI, propose une poésie intitulée «Il giornalista ", dans laquelle un grand reporter, après avoir traversé les cinq continents et les contrées les plus diverses, ramène enfin une nouvelle :

Et sais-tu ce que je ramène?

Une seule nouvelle!

Je vais être licencié pour paresse.

Mais l'événement est sensationnel,

Il mérite un gros titre :

Tous les peuples de la terre

Ont déclaré la guerre à la guerre. ${ }^{60}$ 
Après cet appel antimilitariste, la poésie entrera dans les articles des journalistes. Dans Il était deux fois le baron Lambert, un journaliste anglais qui campe dans les bois admire le lever du soleil sur le Mont-Rose et envoie à son directeur un article décrivant ce spectacle :

Le journaliste a décrit avec enthousiasme ce spectacle dans un article que son directeur a jeté au panier, dictant aussitôt après un télégramme urgent : « Laisse tomber le paysage, les gens ne veulent pas savoir ce que fait le Mont-Rose mais ce que fait le baron Lambert. »

La réaction du directeur sera sans appel et notre journaliste se contentera de composer des poésies pour lui-même. Rodari reprend aussi la technique du renversement qui lui est chère et que nous avons déjà repérée dans Gelsomino... Un texte bref, Scoop, met en scène un tout jeune journaliste qui rapporte dans ses articles des événements qui n'ont pas eu lieu et les narre, à la forme négative, avec une précision du détail qui rend vraisemblable une nouvelle présentée dans la rubrique des faits divers :

« Hier à 14 h 35, sur la nationale 897, il ne s'est pas produit une terrifiante collision dans laquelle n'ont pas perdu la vie cinq personnes.

Un gros semi-remorque immatriculé MI 2345, dans sa tentative de ne pas doubler un tracteur agricole, n'a pas heurté de plein fouet la voiture immatriculée ROMA 4567, qui roulait en sens contraire, et qui par conséquent ne s'est pas renversée sur trois motocyclistes belges survenant à ce moment-là. Dans cet accident ne sont pas morts : un camionneur, deux des motocyclistes, le jeune homme qui conduisait la voiture et sa fiancée ; quant aux autres, ils n'ont pas eu de blessures plus ou moins graves n'imposant donc pas leur transport d'urgence à l'hôpital. La police routière, pour sa part, n'a pas eu à ouvrir d'enquête. $»^{61}$

Il est évident que le directeur du journal ne l'entend pas de la même oreille et convoque son jeune collègue pour le sermonner: « Nous sommes en train de devenir un journal pour fantômes : bientôt nous imprimerons à l'encre blanche!» Le jeune journaliste défend son point de vue en précisant que rien n'est faux dans ses déclarations et essaie d'apporter des fondements théoriques à sa position en se posant la question de ce que devrait être le journalisme moderne, qui doit faire apprécier la vie: "Un journal moderne doit pouvoir élargir son champ d'action au domaine du possible. » La fin est prévisible : il est licencié, pour la quatrième fois, mais continue imperturbable à croire en ses choix : «Il faut croire que mes idées sur le journalisme sont trop avancées. Peu importe, je vais chercher une nouvelle place. Je suis jeune, j'ai tout l'avenir devant moi.»

Ce qui est agréablement paradoxal, c'est que ce sont ces journalistes les plus farfelus que nous voyons réellement travailler et dont nous pouvons lire les productions. Ils peuvent basculer résolument du côté des enfants. Dans la série des «enfantastiques " ${ }^{62}$, M. Bertrand, le journaliste qui habite à côté de l'école de la Contrescarpe, bénéficie d'une aura particulière, car il n'a pas de préjugé négatif et participe aux bêtises des enfantastiques. Avec eux, il s'envole en parapluie au-dessus de Paris, leur commente les monuments survolés, et ne s'étonne pas particulièrement des vêtements qui volent : on pourrait le taxer d'incompétent, mais il a plutôt gardé une capacité à s'émerveiller comme un enfant et à accepter des événements incongrus. Il fait parfois aussi les frais de ces aventures étranges mais ne s'en émeut guère et surtout ne garde aucune rancune. Dans "L'enfant qui effaçait les gens " ${ }^{63}$, une fillette qui possède le pouvoir d'effacer d'un coup d'éponge les gens qui l'ennuient se met tout à coup à gommer ainsi la partie haute des corps. Le journaliste, toujours à l'affût d'un scoop, se rend immédiatement sur les lieux car « [i]l souhait interviewer toutes ces jambes sans corps, 
mais les jambes ne répondaient pas. » [...] «Eh bien quoi ? » demanda-t-il aux jambes. "Ce n'est qu'une fillette... » Il n'eut pas le temps d'en dire davantage. Pfuitt! Plus de journaliste sauf les jambes!»[P.37]. Dans «L'enfant qui faisait disparaitre les vêtements " de Rivais, le journaliste, M. Bertrand, est une fois de plus la cible des enfantastiques. Thomas fait voler sa casquette et le narrateur s'attarde sur une scène digne des meilleurs numéros de clown :

M. Bertrand s'était rendu compte qu'il était tête nue à l'instant où il ouvrait la portière de l'automobile. Il se retourna, se baissa pour regarder sous la voiture.

« Hi-hi-hi ! » ricana Antoine. « Il croit qu'il a provoqué un courant d'air en ouvrant la portière!»

M. Bertrand revenait sur ses pas, regardait derrière une poubelle sur le trottoir. La casquette n'était pas là non plus. [...]

M. Bertrand venait de soulever le couvercle de la poubelle pour y jeter un coup d'œil dedans : mais il n'y trouva pas sa casquette. Il leva les bras en l'air et monta en voiture. [P. 15-16]

70 Les gendarmes finissent par arriver car le quartier est sens dessus dessous et lorsque le journaliste revient pour questionner les enfants sur une éventuelle émeute un simple «Oh non, Monsieur Bertrand!» suffit à le faire renoncer à enquêter, en pensant qu'« On le dérangeait souvent pour rien. » À ce moment-là, il aperçoit deux habits qui flottent en l'air mais ne s'en étonne pas davantage pensant simplement qu'il devra changer ses lunettes, "[p]uis il ajusta sa casquette sur sa tête et remonta dans son automobile puisqu'il ne se passait décidément jamais rien sur la place de la Contrescarpe. » [P. 28] Ne trouvant pas particulièrement étranges tous ces événements, image renversée du journaliste méfiant, suspicieux et à la recherche du sujet, M. Bertrand passe systématiquement à côté du scoop, voire même du simple sujet d'article, mais il garde la confiance des enfants et de la population.

71 Si le nombre de textes où évoluent des journalistes n'est pas très important au regard de la production pour enfants, nous avons vu qu'il nous offre toutefois un large panel de représentations de ce personnage, que celui-ci soit acteur ou témoin, personnage principal ou comparse. Dans un corpus qui concerne trois langues et un demi-siècle, nous voyons se dégager des constantes dans les thèmes abordés et des similitudes de traitement. Des thèmes réputés difficiles, sensibles, réservés aux adultes, comme la presse au service du pouvoir, le harcèlement par les médias et la désinformation sont ici amplement exploités, dans des textes aux caractéristiques narratives et stylistiques bien différentes. Ainsi la question de la dépendance ou soumission de la presse vis-à-vis du pouvoir ou de l'idéologie dominante apparaît-elle dans des textes aussi différents que Cipollino nel paese dei bugiardi, Fantastique Fantômette et Les Reliques de la mort. Si parfois le journaliste apparaît de manière stéréotypée, les images qui sont proposées au jeune lecteur ne sont que rarement simplistes, témoignant par là d'une motivation particulière des écrivains lors de l'élaboration de ces personnages de fiction, qui constituent souvent pour le jeune lecteur un premier contact avec cette profession. Certes l'impact de ces lectures dans la construction du regard que le jeune lecteur portera ensuite sur presse et journalisme reste évidemment impossible à évaluer. En ce qui concerne les techniques narratives et le traitement des personnages, l'influence de Rodari sur Rivais nous semble évidente dans les renversements de nouvelles, l'imagination débridée qui se coule naturellement dans le réel, l'amour de la poésie. Si les facettes les plus noires et contestables de cette profession ne sont pas cachées, il paraît indéniable que les textes humoristiques nous apportent la plus grande richesse d'exploitation de ces personnages imaginaires, en nous offrant des textes 
particulièrement savoureux et un regard porté sur cette profession fréquemment original voire largement investi par une imagination débridée.

\section{NOTES}

1. Si nous nous cantonnons à l'aire francophone, le reporter le plus connu de la bande dessinée est bien entendu Tintin créé en 1929 par Hergé et, parmi les héros récurrents, nous trouvons : Ric Hochet, Guy Lefranc, Jacques Flash, Marc Dacier mais aussi Babiole et Zou de Greg, Jeannette Pointu, Fantasio, l'homme aux multiples métiers qui finit par devenir reporter-photographe, Seccotine, la journaliste d'investigation créée en 1953 et Gaston Lagaffe, le garçon du courrier. Parfois le journaliste n'apparaît que le temps d'un épisode, comme dans Daily star de la série des Lucky Luke, Le schtroumpf reporter. Parmi les seconds rôles figurent le père de l'héroïne dans Marion Duval (Yvan Pommaux, BD publiée dans Astrapi puis chez Bayard éditions jeunesse, Bayard $\mathrm{BD})$. Beaucoup plus rares sont les journalistes héros de papier en Italie : nous trouvons quelques reporters dans l'œuvre de Hugo Pratt (Jack London et Ernie Pike inspiré du reporter Ernest Pyle).

2. Ruth Amossy, Anne Herschberg Pierrot, Stéréotypes et clichés. Langue discours société, s. l.: Armand Colin, 2007, [1 $1^{\text {re }}$ éd. Nathan 1997], p. 37. Voir aussi Ruth Amossy, «Du cliché au stéréotype. Bilan provisoire d'un parcours ", in Gilles Mathis (dir.), Le cliché, Toulouse : Presses Universitaires du Mirail, coll. Interlangues littératures, 1998, p. 21-28; Ruth Amossy, Elisheva Rosen, Les discours du cliché, Paris : CDU et Sedes Réunis, 1982, $151 \mathrm{p}$.

3. Ruth Amossy, Les idées reçues. Sémiologie du stéréotype, Paris : Nathan, Le texte à l'œuvre, 1991, p. 43.

4. R. Amossy a mis en lumière ce point en 1991 dans son ouvrage Les idées reçues. Sémiologie du stéréotype, op. cit., p. 9 : « Aujourd'hui, la presse, la B.D., les best-sellers, le cinéma, la publicité ne cessent de renforcer ou de forger à notre usage des stéréotypes de tout acabit. Réciproquement, la production culturelle se nourrit des images qui circulent dans la société contemporaine. »

5. Malgré cet arc temporel étendu qui nous permet d'aborder des textes particulièrement significatifs, ce travail ne se veut en aucun cas exhaustif.

6. Gianni Rodari (1920-1980) est considéré comme l'un des plus grands auteurs pour la jeunesse, son œuvre a été couronnée en 1970 par le Prix Andersen, plus haute reconnaissance en littérature de jeunesse. Un regard porté uniquement sur la diffusion de son œuvre en France pourrait être trompeur: alors que chez nous il reste encore presque exclusivement connu des spécialistes de littérature de jeunesse et des enseignants, du primaire notamment, en Italie ses livres ont été largement diffusés et aujourd'hui encore les rééditions ou éditions nouvelles, avec parfois de rares inédits, sont fréquentes.

7. Yak Rivais, Pas de panique!, Paris : L'Ecole des Loisirs, Neuf en poche, 1986. Né en 1939, Yak Rivais a mené parallèlement à sa carrière d'instituteur une activité foisonnante comme peintre et dessinateur humoristique, écrivain mais surtout auteur pour la jeunesse, avec une prédilection pour les contes et nouvelles où dominent fantastique et jeux de mots.

8. Claude Bourgeyx, «Riton-La-Malice ", in Le fil à retordre, Paris : Nathan, Nathan Poche 10-12 ans, Humour, ill. de Serge Bloch et Bruno Jarret, p. 35-36. Claude Bourgeyx, né en 1943, est romancier, nouvelliste, dramaturge, scénariste, dialoguiste. Pour les enfants, il a publié plusieurs ouvrages remarquables pour leur humour teinté de poésie (voir Olivier Poncer, Claude Bourgeyx, 
Une histoire à dormir, s. 1. : Thierry Magnier, 1998 ; Claude Bourgeyx, Jochen Gerner, Grosses têtes et petits pieds, 28 histoires impertinentes, Paris : Nathan, 2002, 150 p.).

9. Concernant ce personnage, voir Armelle Leroy, Laurent Chollet, Le Club des Cinq, Fantômette, Oui-Oui et les autres. Les grands succès de la Bibliothèque rose et verte, Hors-Collection, 2005 ; Pierre Bannier, Les micro-sociétés de la littérature pour la jeunesse. L'exemple de Fantômette, Paris: L'Harmattan, Logiques sociales, 2000, 187 p. Un site non officiel mais fort documenté se révèle aussi riche en informations : [En ligne].

10. Né en 1931, Georges Chaulet suit des études d'architecture mais décide rapidement d'écrire pour les enfants. Entre 1958 et 1962, il publie chez Casterman la série "Les 4 As » avant de donner naissance à Fantômette, héroïne spécifiquement créée pour les filles, dont Chaulet sait qu'elles lisent davantage que les garçons. Les Exploits de Fantômette sont publiés en 1961, suivront 48 titres jusqu'en 1987. Un 50 titre sera écrit en 2006 pour fêter les 150 ans de la Bibliothèque Rose.

Georges Chaulet, Fantômette et le brigand, Paris : Hachette, Bibliothèque Rose, 2002 [1 $1^{\mathrm{re}}$ éd. 1968], 152 p. ; nous citerons cet ouvrage sous la référence [FB] ; Fantômette et le trésor du pharaon, Paris : Hachette, Bibliothèque Rose, 2002 [1 $1^{\text {re }}$ éd. 1970], 153 p. ; Fantômette viendra ce soir, Paris : Hachette, Bibliothèque Rose, 2003 [1 $1^{\mathrm{re}}$ éd. 1976], 218 p.; Mission impossible pour Fantômette, Paris : Hachette, Bibliothèque Rose, 2000 [ $1^{\text {re }}$ éd. 1977], 152 p. ; nous citerons cet ouvrage sous la référence [MIPF]; Fantastique Fantômette, Paris : Hachette, Bibliothèque Rose, 2000 [1 $1^{\text {re }}$ éd. 1978] [à partir de 9 ans].

11. Nous ajouterons par ailleurs quelques remarques à partir de la série Esprit fantômes écrite par Didier Julia et Valérie Hadida (Paris : Hachette, Bibliothèque Rose, à partir de 7 ans).

12. Le premier volume des aventures de Geronimo Stilton a été publié en Italie chez l'éditeur Piemme; en 2008 plus de 70 aventures ont vu le jour, sans compter les ouvrages dérivés. Ce personnage a été imaginé par Elisabetta Dami dont le nom n'apparaît pourtant pas sur les couvertures : la fiction (et le marketing) veulent que Geronimo Stilton soit l'auteur des histoires narrées. Par ailleurs, il est fort probable qu'aujourd'hui des équipes entières de rédacteurs collaborent à la création de ces ouvrages, même si E. Dami déclare dans ses interviews les écrire en une dizaine de jours, parfois dans un hôtel de Sharm-El-Sheik. Traduit dans 40 langues, publié dans plus de 180 pays dont la France (Albin Jeunesse), Geronimo Stilton a largement dépassé les 10 millions d'exemplaires vendus en Italie et a reçu en 2001 le prix Andersen comme personnage de l'année.

13. Flavio Sasso, Isabella Abate (ill.), Minaccia alla città, [Legnano] : CreaLibri, I giallissimi per ragazzi, 1998, 59 p.; nous citerons cet ouvrage sous la référence [MC]; Massimiliano Francia, Delitto all'ippodromo, Legnano : EdiCart, CreaLibri, 2000, 59 p.

14. Carlo Ossola, Senso di giustizia, Trieste : Edizioni EL, I Corti, 1999, 47 p.

15. J.K Rowling a publié le premier tome des aventures du jeune sorcier Harry Potter sous le titre Harry Potter and the Philosopher's Stone en 1997, London : Bloomsbury Publishing, 1997 ; éd. française : Harry Potter à l'école des sorciers, Paris : Gallimard jeunesse, 1998, trad. de J.-F. Ménard. La saga s'est achevée en 2007 avec la publication du $7^{\mathrm{e}}$ et dernier tome Harry Potter and the Deathly Hallows ; London : Bloomsbury Publishing, 2007 ; éd. française Harry Potter et les reliques de la mort, Paris : Gallimard, 2007, 809 p., trad. de J.-F. Ménard.

16. Nous renvoyons notamment notre lecteur à l'analyse du roman Paris-Bagdad d'olivier Ravanello (2007, Grasset Jeunesse) par Jean Perrot, Mondialisation et littérature de jeunesse, IX Féminin-masculin : de la guerre des peuples au jeu de la personne dans l'institution (tragédies, comédies, fantaisies), s. 1.: Editions du Cercle de la Librairie, Bibliothèques, 2008, 381 p. Par ailleurs, pour des questions de temps, nous privilégions ici le journaliste de presse écrite, mais il serait extrêmement pertinent d'articuler cette analyse avec celle de la représentation du journaliste de télévision.

17. Publié en 1907, le Mystère de la chambre jaune, suivi l'année suivante du Parfum de la Dame en noir, fit de Joseph Rouletabille, jeune reporter à L'Epoque, l'archétype du journaliste 
d'investigation. Si aujourd'hui ces œuvres sont publiées dans des éditions explicitement destinées à la jeunesse et parfois données à lire dès le primaire, elles ne furent pourtant pas écrites pour ce jeune public. En ce qui concerne ce personnage, nous renvoyons notamment notre lecteur à Jacques Dubois, Le roman policier ou la modernité, chap. IX L'utopie de Rouletabille,

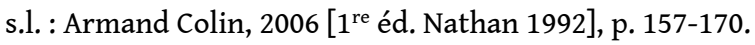

18. Dans ce choix de textes, seul Geronimo Stilton est un protagoniste présenté comme le héros des aventures; dans les autres ouvrages, les journalistes peuvent prendre un rôle de premier plan le temps d'un épisode, d'une nouvelle, voire de quelques chapitres ou même seulement d'un passage. Dans quelques textes enfin, ils n'apparaissent que de manière indirecte.

19. Instituteur, Gianni Rodari quitte cette profession après un petit nombre d'années d'exercice pour embrasser celle de journaliste, tout d'abord auprès de publications locales, puis comme pigiste à l'édition milanaise de l'Unità, organe du Parti communiste italien : c'est là qu'en 1949, il commence à écrire "presque par hasard», comme il aimera le souligner, des poésies et comptines pour enfants. Il mène jusqu'à sa mort une double carrière d'écrivain pour enfants et de journaliste, à l'Unità de Milan et de Rome où il s'occupe de questions d'actualité, avant de devenir envoyé spécial et billettiste, puis à Paese Sera où il couvre des sujets de société, de politique mais aussi de littérature, d'éducation et de pédagogie. Il dirigea plusieurs périodiques pour enfants ou adolescents (La Via, Il Pioniere) ainsi que des revues destinées aux parents (Il Giornale dei Genitori). Malheureusement, son activité de journaliste reste quasiment inconnue du public français.

20. Gianni Rodari, Jip dans le téléviseur, s. 1.: Scandéditions / La Farandole en poche, 1993, ill., trad. A. Monjo, 89 p. ; [titre original Gip nel televisore, Milano : Mursia, 1976, ill. [à partir de 8 ans] ; Gelsomino nel paese dei bugiardi, Roma : Editori Riuniti, 1987 [1a ed. 1971], ill. Raoul Verdini, 158 p. ; Gianni Rodari, Il était deux fois le baron Lambert, [Genève] : La joie de lire, 2000, 188 p. [traduit de l'italien par Roger Salomon, titre original C'era due volte il Barone Lamberto, Torino : Einaudi, 1978] ; Gianni Rodari, Scoop, traduction adaptation de Roger Salomon, Rue du Monde, coll. La Maisonaux-histoires, 1999, ill. Pef. Texte adapté à partir de « Un benefattore incompreso », l'Unità, 18 août 1957, publié in Il cane di Magonza, Roma : Editori Riuniti, 1982, p. 43-46.

21. "O giornalista inviato speciale / quali notizie porti al giornale ? », « Il giornalista », in « Le filastrocche dei mestieri », Filastrocche in cielo e in terra, Torino : Einaudi, p. 88. Sauf mention de traducteur dans la référence bibliographique, nous sommes l'auteure des traductions proposées.

22. «Il refuso è quella cosa / che tu trovi nel giornale / e ci resti molto male/ se non sei svelto a capir / [...] / Il refuso in conclusione / è il burlone del giornale / e può far sorgere il sale / mentre noi s'aspetta il sol. », comptine publiée in Il giornale dei genitori au début des années soixante-dix, reprise in " Notizie di cronaca », Filastrocche per tutto l'anno, ill. E. Luzzati, Roma : Editori Riuniti, 1986, p. 80.

23. Gianni Rodari, Il était deux fois le baron Lambert, op.cit. Nous citerons cet ouvrage sous la référence [BL].

24. "I gatti hanno un giornale / Con tutte le novità / E sull'ultima pagina / La 'Piccola pubblicità'. ", « Il giornale dei gatti », in Gelsomino nel paese dei bugiardi, Roma : Editori Riuniti, 1987 [1a ed. 1971], ill. Raoul Verdini, 158 p., « Le canzoni di Gelsomino », p. 140-141.

25. Ce texte a été publié en France sous le titre Benjamin au pays des menteurs, Paris : Hachette, Bibliothèque Rose, 1980, 155 p., trad. Candido Temperini. Nous citerons cet ouvrage sous la référence $[\mathrm{CPB}]$ et proposons les traductions.

26. Respectivement intitulés The Daily Prophet et The Quibbler dans la version anglaise.

27. J.K. Rowlings, Harry Potter et les Reliques de la Mort, op. cit. Nous citerons cet ouvrage sous la référence $[\mathrm{RM}]$.

28. Flavio Sasso, Isabella Abate (ill.), Minaccia alla città, op. cit. 
29. «Scoop era magro e allampanato, talmente alto che le giacche gli cascavano dalle spalle come le pieghe di un tendaggio. Assomigliava ad un attaccapanni a stelo con tanto di eterno farfallino multicolore. "

30. Sur les personnages d'enquêteurs, voir Yves Reuters, Le roman policier, Paris : Lettres 128,

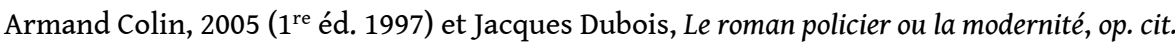

31. «reporter di cronaca nera e mio grande amico. Ci scambiavamo spesso notizie utili e interessanti per entrambi ; cosa, nel nostro mestiere, molto preziosa. »

32. Massimiliano Francia, Delitto all'ippodromo, op. cit.

33. «Tim aveva iniziato la sua carriera come fattorino presso un giornale, proprio alla Gazzette. La sua intelligenza, la sua tenacia di mastino e la sua astuzia da volpone esperto, unite a una grande intuizione nello scovare le notizie più sensazionali partendo da piccoli indizi, lo avevano portato in breve tempo a salire nella considerazione dei proprietari del giornale; gradino dopo gradino aveva fatto una splendida carriera, ed era salito ai piani alti del palazzo, quelli che contano ! E sia chiaro : soltanto e unicamente per i suoi meriti. / Il suo ufficio era vicino a quello del direttore.»

34. «Pochi conoscevano come lui le porcherie e gli intrighi che si nascondevano [...]. »

35. «[...] il rischio di farsi impiombare per la verità di cronaca lo metteva in agitazione, senza però intimidirlo. "

36. «[...] [la] sua durissima battaglia, che aveva le proporzioni, ogni giorno di più, di una gigantesca quanto impossibile crociata contro il crimine. »

37. «I giornalisti fecero appena in tempo a infilarsi la penna stilografica nel taschino e a saltare sulla loro automobile. »

38. A son tour, Tea Stilton, enseignant par ailleurs le journalisme, écrit maintenant des livres dans lesquels elle raconte les aventures des "Tea sisters", groupe de cinq jeunes camarades détectives.

39. « Un'ora più tardi uscì un'edizione straordinaria del Perfetto bugiardo. »

40. Les italiques sont présentes dans l'édition française.

41. "Giornalisticamente deludente ».

42. «L'inchiesta si tirò avanti verso una prevedibile archiviazione. Sui giornali, come al solito, gli articoli rimpicciolirono nelle pagine interne, giorno dopo giorno, fino a sparire ».

43. Jacques Dubois, op. cit, p. 167.

44. «Salve Rex, proprio adesso mi stavo apprestando a mandare in stampa l'edizione della sera ; non ho tempo ora. ", p. 19.

45. «La sua scrivania era occupata soltanto da tre telefoni, con i quali dettava gli articoli e svolgeva la maggior parte del suo lavoro: che consisteva infine nel mantenere contatti con mezzo mondo. »

46. "[...] mi piaceva il suo modo di lavorare : gli articoli che scriveva facevano più male ai delinquenti di ogni risma che una scarica di piombo. Aveva, secondo me, un gran coraggio ; già, perché, se non lo sapete, le battaglie non si vincono soltanto con le pistole, ma occorre un buon cervello!»

47. «Tim, detto «Scoop » per la sua abilità nello scovare le informazioni più segrete, aveva publicato una serie di articoli sulla Gazzette, il quotidiano più indipendente e venduto della città, denunciando il possibile legame tra la malavita, il mondo degli affari e la politica./ Certo è che sapeva davvero molte cose sulla nostra amatissima e luridissima metropoli e sui suoi affari loschi... Ma quello che forse preoccupava è che molte di queste cose non le diceva né le scriveva!»

48. Claude Bourgeyx, « Riton-La-Malice », Le fil à retordre, op. cit.

49. «[...] per scovare le notizie più strane, usando la sua simpatia e qualche dollaro che passava di mano.» 
50. «Introvabile e invisibile, lo aiutava la sua agenda telefonica ultrasegreta, il suo strumento di lavoro più prezioso. Si diceva che vi erano segnati i numeri telefonici privati dei personaggi più potenti della nazione!»

51. Il giornale si intitolava: Il perfetto bugiardo, e naturalmente era fatto tutto di notizie false o di fatti veri raccontati a rovescio./ C'era per esempio un articolo intitolato : « Una grande vittoria del corridore Persichetti». Ecco il testo della notizia : "Il noto campione di corsa nei sacchi Flavio Persichetti ha vinto ieri la decima tappa del giro del Regno, distaccando di venti minuti Romolo Baroni, giunto secondo, e di trenta minuti e quindici secondi Piero Clementini giunto terzo. Il gruppo, arrivato un'ora dopo il vincitore, è stato battuto in volata da Pasqualino Balsimelli ».

52. «Mancata tragedia sulla via Cornelia - Cinque persone non muoiono e dieci altre non riportano la minima ferita ».

53. "Come il lettore può vedere con le sue orecchie, non è successo assolutamente nulla ».

54. «Non è assolutamente vero che la polizia stia ricercando il noto tenore Gelsomino. Non ce ne sarebbe nessuna ragione, perché Gelsomino non deve affatto rispondere dei danni che non ha causato al Teatro Comunale. Perciò, chiunque sappia dove Gelsomino si nasconde non lo vada dire alla polizia, perché sarebbe rimproverato severamente. »

55. - Ha niente da dichiarare al Perfetto bugiardo ? gli domandarono i giornalisti./ - Miao, rispose Calimero voltando le spalle./ - Ottimamente, dissero i giornalisti. - Lei è uno dei testimoni oculari. Può dirci come mai non è successo niente?/ - Miao, - fece ancora Calimero./ Benissimo. Smentiremo nel modo più assoluto che il manicomio sia crollato e che i pazzi si siano sparsi per la città./ - Ma la volete capire, sbottò Calimero, la volete capire sì o no che io sono un gatto ?/ - Vorrà dire un cane, dal momento che miagola.

56. Denis Côté, Frédéric Rébéna (ill.), Les aventures de Jos Tempête. La machination du Scorpion noir, Paris : Nathan, Lune Noire, Fantastique, 2001, p. 43.

57. Ibidem, p. 56.

58. Gianni Rodari, Jip dans le téléviseur, Scandéditions / La Farandole en poche, 1993, ill., trad. A. Monjo, 89 p.; [titre original Gip nel televisore e altre storie in orbita, Milano: Mursia, 1976, ill. 157 p.], [à partir de 8 ans]. Nous citerons ce texte avec la référence [JT].

59. "Stanno succedendo cose grosse, signor direttore. La gente ci ha riso in faccia e ci ha consigliato di adoperare il nostro giornale per farci delle barchette di carta. »

60. "e sai che porto?/ una sola notizia !/ Sarò licenziato per pigrizia./ Però il fatto è sensazionale,/ merita un titolo cubitale:/ tutti i popoli della terra/ han dichiarato guerra alla guerra. », " Il giornalista », " Le filastrocche dei mestieri », in Filastrocche in cielo e in terra, Torino : Einaudi, p. 88.

61. Scoop, Traduction adaptation de Roger Salomon, Rue du Monde, coll. La Maison-auxhistoires, 1999, ill. Pef. Texte adapté à partir de "Un benefattore incompreso ", l'Unità, 18 août 1957, publié in Il cane di Magonza, Roma : Editori Riuniti, 1982, p. 43-46.

62. Les " enfantastiques" sont des enfants du quartier de la Contrescarpe à Paris, rue MarcelAymé, dotés de pouvoirs extraordinaires. Leurs aventures sont narrées dans une quinzaine de titres parus à L'Ecole des Loisirs et le recueil Bonjour les enfantastiques (L'Archipel, 2007).

63. «L'enfant qui effaçait les gens », op. cit., p. 31-39. 


\section{RÉSUMÉS}

À partir d'un corpus multilingue et multigenre (romans, romans policiers, nouvelles, poésies, comptines), nous souhaitons dégager l'image du journaliste telle qu'elle apparaît dans la littérature de jeunesse des années cinquante à nos jours, en France et Italie, avec un excursus dans la littérature anglaise. Au-delà des représentations, il s'agira notamment de faire émerger les fonctions du journaliste tant au niveau de l'histoire que de la narration. Dépassant l'image stéréotypée du journaliste de fiction, détective intrépide, nous voyons apparaître tout à la fois des reporters à la solde du pouvoir et d'étonnants journalistes entre fantaisie et poésie.

Based in a variety of languages and writings (novels, detective fiction, short stories, poetry and nursery rhymes), this paper analyses the image of the journalist as represented in children's literature from the fifties to today, in France and Italy, with a brief reference to English literature. It analyses the functions of the journalist and his writing both at the level of the story and the narrative. Seeking to explore beyond the stereotype of the fictional journalist as a fearless detective, it examines alternative representations, ranging from reporters subordinated to power to journalists situated between fantasy and poetry.

\section{INDEX}

Mots-clés : littérature de jeunesse, littérature contemporaine, littérature italienne, littérature française, détective, journaliste, Chaulet Georges, Rivais Yak, Rodari Gianni, Rowling J. K., Fantômette

Keywords : Children's literature, contemporary literature, Italian literature, French literature, detective, journalist, Chaulet Georges, Rivais Yak, Rodari Gianni, Rowling J. K., Fantômette

\section{AUTEUR}

\section{SYLVIE MARTIN-MERCIER}

Université Stendhal-Grenoble 3

ILCEA / GREMUTS 